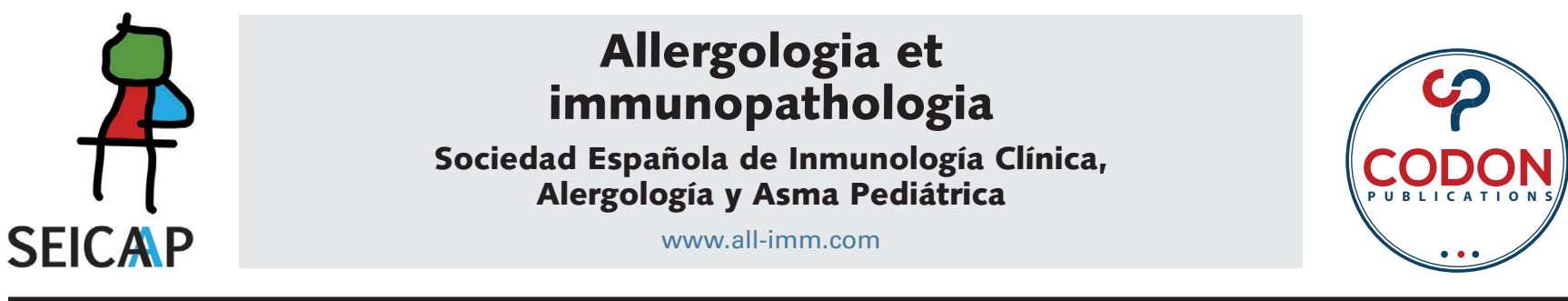

ORIGINAL ARTICLE

\title{
Fish allergy tolerance 16 months after diagnosis
}

\author{
Mitsuhiro Okamoto ${ }^{a *}$, Satoru Takafujia, Shintaro Inoue ${ }^{a}$, Yuya Tanakab
}

${ }^{a}$ Department of Pediatrics, Hyogo Prefectural Tamba Medical Center, Tamba, Japan

${ }^{b}$ Department of Allergy, Hyogo Prefectural Kobe Children's Hospital, Kobe, Japan

Received 13 March 2021; Accepted 14 July 2021

Available online 1 September 2021

\author{
KEYWORDS \\ food hypersensitivity; \\ immunoglobulin $\mathrm{E}$ \\ (IgE); \\ rosefish; \\ jack mackerel; \\ Sebastes; \\ Trachurus; \\ parvalbumin; \\ oral food challenge
}

\begin{abstract}
Fish allergy is generally thought to be persistent, and approximately $80 \%$ of patients with fish allergies do not develop tolerance even 10 years after diagnosis. There have been no reports of rapid tolerance development in patients with severe fish allergies. We report the development of tolerance 16 months after the diagnosis of fish allergies. A 13-month-old boy was diagnosed with rosefish allergy (Sebastes matsubarae) and Japanese jack mackerel allergy (Trachurus japonicus). To find out which species of fish he could consume safely, he underwent several oral food challenge (OFC) tests. It was determined that he could consume tuna, salmon, cod, sardine, chub mackerel (Scomber japonicus), and Japanese amberjack (Seriola quinqueradiata) without eliciting signs of allergy. He continued to eat the fish that did not produce allergic reactions three to four times a week. The titer of serum allergen-specific immunoglobulin E (IgE) to fish had decreased in a subsequent ImmunoCAP ${ }^{\circledR}$-specific IgE blood test performed 16 months after the diagnosis of the rosefish allergy. Following this test result, he underwent OFCs with rosefish and Japanese jack mackerel, both of which turned out to be negative, and it was determined that he had developed tolerance to fish. In this case, the repeated OFCs were useful in identifying fish species that were safe for consumption. In addition, the decrease in allergen-specific IgE was useful in predicting the development of tolerance. We hypothesize that proactive consumption of available fish species may lead to this rapid induction of tolerance to fish allergens.
\end{abstract}

(c) 2021 Codon Publications. Published by Codon Publications.

\section{Introduction}

Fish allergy is one of the most important causes of severe acute hypersensitivity reactions, including fatal anaphylaxis. ${ }^{1}$ The prevalence of fish allergy is estimated to be less than $0.3 \%$ globally. ${ }^{2}$ Fish allergy is generally thought to be persistent, and approximately $80 \%$ of patients with fish allergies do not develop tolerance even 10 years after diagnosis. ${ }^{3}$ There have been no reports of rapid tolerance development in patients with severe fish allergies. This

*Corresponding author: Mitsuhiro Okamoto, MD, Department of Pediatrics, Hyogo Prefectural Tamba Medical Center, Tamba, Japan. Email address: m-okamoto@hp.pref.hyogo.jp 
article reports the development of tolerance 16 months after diagnosis in a pediatric patient allergic to rosefish (Sebastes matsubarae) and Japanese jack mackerel (Trachurus japonicus).

\section{Case report}

A 13-month-old boy was brought to the emergency room with facial erythema and edema, urticaria around the mouth and on the extremities, and stridor, 15 min after ingesting $20 \mathrm{~g}$ of rosefish for the first time at his nursery school. He had a history of atopic dermatitis, especially around the mouth. He had no history of asthma, allergic rhinitis, or other food allergies. On arrival, the patient was conscious, with percutaneous oxygen saturation $\left(\mathrm{SpO}_{2}\right)$ at $90 \%$ on room air and heart rate of 204 bpm (beats per minute). Peripheral pulse was weak compared to centrally palpated pulse. He was diagnosed with anaphylaxis and given an intramuscular injection of $0.01 \mathrm{mg} / \mathrm{kg}$ adrenaline. He was also given intravenous injections of $1 \mathrm{mg} / \mathrm{kg}$ prednisolone and $0.1 \mathrm{mg} / \mathrm{kg}$ d-chlorpheniramine maleate. Nineteen minutes later, a second dose of adrenaline was administered at $0.01 \mathrm{mg} / \mathrm{kg}$, and his respiratory status and vital signs improved. He was diagnosed with rosefish allergy, and fish was subsequently excluded from his diet, except canned tuna and small amounts of sardines, which he had been able to eat without adverse reactions previously. To find out which species of fish he could consume safely, he underwent several oral food challenge (OFC) tests. It was determined that he could consume tuna, salmon, cod, sardine, chub mackerel (Scomber japonicus), and Japanese amberjack (Seriola quinqueradiata) without eliciting signs of allergy. However, he developed perioral urticaria and intermittent coughing during an OFC with $10 \mathrm{~g}$ of Japanese jack mackerel 2 months after the diagnosis of his rosefish allergy. Hence, he was also diagnosed with Japanese jack mackerel allergy. He continued to eat those fish that did not produce allergic reactions three to four times a week. The titer of serum allergen-specific immunoglobulin $\mathrm{E}$ (IgE) to fish had decreased in a subsequent ImmunoCAP ${ }^{\circledR}$-specific IgE blood test performed 16 months after the diagnosis of the rosefish allergy (Figure 1). In contrast, the total IgE levels increased from 177 to $759 \mathrm{lU} / \mathrm{mL}$.

Following this test result, he underwent OFCs with rosefish and Japanese jack mackerel, both of which turned out to be negative. The patient continued eating all of the tested fish species at home, and it was determined that he had developed tolerance to fish.

\section{Discussion}

This case highlights the development of fish tolerance 16 months after the initial diagnosis of fish allergy. In previous reports, it took approximately 10 years to develop tolerance to fish. ${ }^{4}$ Another report indicated that tolerance developed approximately 60 years after the diagnosis. ${ }^{5}$ The present article is the first to report early tolerance in severe fish allergy.

The fact that tolerance may develop early means that performing OFCs to confirm remission is important, even

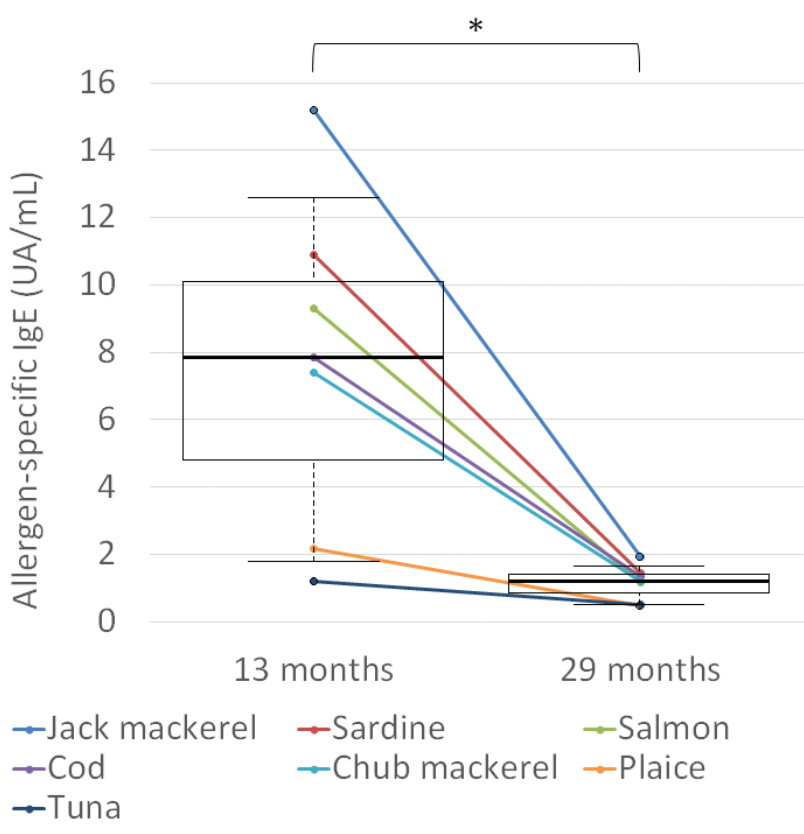

Figure 1. Trends in the fish allergen-specific immunoglobulin E (IgE) levels. Specific IgE reactivity to all fish allergens at diagnosis decreased significantly after 16 months. ${ }^{*} \mathrm{p}<0.05$, Wilcoxon signed-rank test.

for allergies that have historically been considered to be persistent, such as fish allergies. However, OFCs for patients with severe fish allergy symptoms are associated with high risks, including fatal anaphylaxis. In this case, the decrease in allergen-specific IgE predicted tolerance. Although we could not measure the rosefish-specific IgE levels, we could predict that he developed tolerance to the rosefish allergy because the specific IgE levels for all measured fish were reduced. This is consistent with previous reports. ${ }^{4}$ Repeating the allergen-specific IgE test to confirm a declining trend enabled us to perform the OFCs at the appropriate time.

As mentioned above, this patient's allergies were restricted to rosefish and Japanese jack mackerel. This may be associated with the fact that the structure of parvalbumin, which is one of the major proteins in fish, varies among different species. It shares structural homologies ranging from approximately $60 \%$ to $80 \%$ across different species of fish. ${ }^{3}$ We used the Basic Local Alignment Search Tool to search for the homology of parvalbumin between different fish species. ${ }^{6}$ The parvalbumin homologies of yellowfin tuna (Thunnus albacares), Chum salmon (Oncorhynchus keta), Atlantic cod (Gadus morhua), Japanese sardine (Sardinops melanostictus), Chub mackerel, Greater amberjack (Seriola dumerili), Japanese rockfish (Sebastes inermis), and Golden redfish (Sebastes marinus) to Japanese jack mackerel were $75.00 \%$, 78.82\%, 79.63\%, 69.72\%, 84.26\%, 86.24\%, 74.07\%, and $77.27 \%$, respectively. Though data regarding the amino acid sequence of the parvalbumin in rosefish are scarce, the homology of Japanese rockfish and Golden redfish, which are the same genus as the rosefish, to Japanese jack mackerel were not high. Based on the aforementioned, the parvalbumin homologies may not be useful in predicting 
whether a patient who has allergy to one fish can eat another fish. In this case, repeating the OFC test was useful in searching for fish species that could be ingested.

We believe that it is significant that the patient in this case was able to safely consume fish species other than the ones he was allergic to. The rapid tolerance development seen in this case may have been due to the proactive consumption of fish species that he tested negative for in follow-up OFCs. Active consumption of safe fish species may serve as a type of oral immunotherapy. If this hypothesis is correct, it is quite important to identify fish species that patients with fish allergies are able to consume. However, this can be challenging to achieve. A study estimated that $50 \%$ of patients with a fish allergy show an allergic reaction to at least one other type of fish. ${ }^{7}$ In other reports, only $41 \%-44 \%$ of patients with a fish allergy were able to consume at least one other type of fish. ${ }^{8,9}$ Allergen-specific IgE blood tests may be useful for predicting fish allergies; however, an OFC is still necessary for a reliable diagnosis.

The diagnosis of fish allergy requires the exclusion of scombroid poisoning and anisakis allergy. It is known that several types of dark meat fish (e.g., tuna) contain these histamine-like compounds; however, rosefish is not a dark meat fish. Furthermore, other children at the patient's nursery school also ate the rosefish, but he was the only one who developed symptoms. This suggests that scombroid poisoning was unlikely. The patient in this case was not tested for anisakis allergen-specific IgE, but this test can be used for the diagnosis of anisakis allergy..$^{10}$ Nevertheless, it is unlikely that he would be allergic to anisakis because he was able to continue eating Salmon, Sardine, and Chub mackerel, all of which are prone to anisakis parasites.

\section{Conclusion}

In conclusion, we report the development of tolerance 16 months after the diagnosis of fish allergies. The allergies were restricted to rosefish and Japanese jack mackerel, and the repeated OFCs were useful in identifying fish species that were safe for consumption. In addition, the decrease in allergen-specific IgE was useful in predicting the development of tolerance. We hypothesize that proactive consumption of available fish species may lead to this rapid induction of tolerance to fish allergens.

\section{References}

1. Bock SA, Munoz-Furlong A, Sampson HA. Fatalities due to anaphylactic reactions to foods. J Allergy Clin Immunol. 2001: 107: 191-193. https://doi.org/10.1067/mai.2001.112031

2. Moonesinghe $\mathrm{H}$, Mackenzie $\mathrm{H}$, Venter $\mathrm{C}$, Kilburn S, Turner $\mathrm{P}$, Weir $\mathrm{K}$, et al. Prevalence of fish and shellfish allergy: A systematic review. Ann Allergy Asthma Immunol. 2016; 117: 264-272. https://doi.org/10.1016/j.anai.2016.07.015

3. Tsabouri S, Triga M, Makris M, Kalogeromitros D, Church MK, Priftis KN. Fish and shellfish allergy in children: Review of a persistent food allergy. Pediatr Allergy Immunol. 2012; 23: 608-615. https://doi.org/10.1111/j.1399-3038.2012.01275.x

4. Carvalho S, Marcelino J, Cabral Duarte MF, Costa C, Barbosa MA, Pereira Dos Santos MC. Role of recombinant parvalbumin Gad c 1 in the diagnosis and prognosis of fish allergy. J Investig Allergol Clin Immunol. 2020; 30: 340-345. https:// doi.org/10.18176/jiaci.0437

5. Solensky R. Resolution of fish allergy: A case report. Ann Allergy Asthma Immunol. 2003; 91: 411-412. https://doi. org/10.1016/S1081-1206(10)61690-0

6. Open database: Basic Local Alignment Search Tool [Internet]. Bethesda, (MD): National Center for Biotechnology Information (US); c1988 - [cited 2021 Feb 8]. Available from: https://blast. ncbi.nlm.nih.gov/Blast.cgi.

7. Sicherer SH. Clinical implications of cross-reactive food allergens. J Allergy Clin Immunol. 2001; 108: 881-890. https://doi. org/10.1067/mai.2001.118515

8. Aas K. Studies of hypersensitivity to fish. A clinical study. Int Arch Allergy Appl Immunol. 1966; 29: 346-363. https://doi. org/10.1159/000229716

9. Schulkes KJ, Klemans RJ, Knigge $L$, de Bruin-Weller $M$, Bruijnzeel-Koomen CA, Marknell deWitt A, et al. Specific IgE to fish extracts does not predict allergy to specific species within an adult fish allergic population. Clin Transl Allergy. 2014; 4: 27. https://doi.org/10.1186/2045-7022-4-27

10. de Las Vecillas L, Muñoz-Cacho P, López-Hoyos $M$, Monttecchiani V, Martínez-Sernández V, Ubeira FM, et al. Analysis of Ani s 7 and Ani s 1 allergens as biomarkers of sensitization and allergy severity in human anisakiasis. Sci Rep. 2020; 10: 11275. https://doi.org/10.1038/s41598-020-67786-w 